\title{
Entamoeba coli as probable risk factor for women sub-fertility
}

\begin{abstract}
Entamoeba coli is intestinal parasitic microorganism which is regarded tell now as commensal amoeba although their adverse clinical manifestations to the patients infected with it. It altered the homeostasis of the body, by disturbing levels of vital electrolytes such as phosphorus and calcium and also leads to elevation of cholesterol level. Alterations of these vital elements affect the fertility of women and men. Heavy studies are required to exclude or confirm this theory about the harmful effect of Entamoeba coli infection in reproductive status of both partners'. Author is looking for fund to prove his theory about Entamoeba coli, in order to reclassify it from commensal to pathogenic amoeba.
\end{abstract}

Keywords: entamoeba coli, infection, fertility, vital elements, electrolytes, phosphorus, calcium, entamoeba histolytica, karyosome, trophozoite, precystic, glycogen vacuoles
Volume 8 Issue $6-2019$

\author{
Mosab Nouraldein Mohammed Hamad \\ Department of Medical Parasitology, Faculty of Health Science, \\ Elsheikh Abdallah Elbadri University, Sudan
}

Correspondence: Mosab Nouraldein Mohammed Hamad, Department of Medical Parasitology, Faculty of Health Science, Elsheikh Abdallah Elbadri University, Sudan, Tel +249929194137, Email muab.nor13@gmail.com

Received: November 02, 2019 | Published: November 26 2019

\section{Article}

Entamoeba coli is viewed up to date as a commensal species of Entamoeba that frequently exists as non pathogenic parasite in the human gastrointestinal tract, certainly in inhabit the large bowel. It is important in medicine since it can be puzzled throughout microscopic inspection of stained stool samples with Entamoeba histolytica. As this bias is typically done by visual inspection of the parasitic cysts via light microscopy, new methods with molecular biology methods have been developed. ${ }^{1}$ Entamoeba coli is a multi-ethnic in sharing and has been confirmed to arise in about $50 \%$ of human population. It's a monogenetic life form. Three different morphological shapes survive throughout the life cycle vegetative form, Pre-cyst and Cyst.

Vegetative form is about 20 to 30 in diameter with diversity from 10 to 50. It is unicellular. The cytoplasm is differentiated into external thin ectoplasm which is not so prominent and internal granular, vacuolated endoplasm containing bacteria and debris inside food vacuoles. A single nucleus lies inside the endoplasm. It is a ring like arrangement with thick nuclear membrane lined with unequally distributed masses of chromatin and a large, irregular, eccentric karyosome. ${ }^{1}$ Fine linin threads develop between nuclear membrane and karyosome. Active form bears one too numerous pseudopodia which are dumpy, blunted and granular. Motility is sluggish and commonly not directional. The trophozoite nourishes upon bacteria, vegetable cells and other faecal debris exist in the large intestine. Dobell (1938) reported that it may probably ingest R.B.C, rarely. It reproduces by binary fission.

The active form alters into spherical uninucleate precystic stage. The precystic stage size ranges from 15 to $45 ц$ in diameter. It is like to trophozoite stage, except that it is non feeding stage and for this reason food inclusions are not present in the endoplasm. Precystic stage develops into cystic stage upon the unfavorable conditions. The cysts are spherical with size ranging from 10 to $33 \mu \mathrm{m}$ in diameter. Its wall is thick. Immature, it may have one-two or four nuclei with eccentric karyosome .Rarely; it may bear 16 or even 32 nuclei. Glycogen vacuoles and chromatid bodies are seen in the endoplasm up to binucleate stage after that they are consumed. Matured cyst is the infective stage. It formed in the large bowel is discharged out o the host's body through faeces. The cysts live for 3-4 months exterior the body of the host and are moderately more resistant to dryness as compared to those of E. histolytica. The live rate of the cyst is about $46 \%$.

Infectivity to the new host occurs by eating impure food and drinks. The infective cysts are passed from faces to the food items through arthropods and rodents. In the small intestine of the new host excystation occurs through which a single multinucleate amoeba comes out through the cyst wall. It divides into as a lot of immature amoebas as there are nuclei in the cyst. ${ }^{1}$ E. coli resides within the lumen of the large bowel in human. They never go into the mucosa or sub-mucosa layers or other tissues of the intestine. There is no reliable proof that it ever produces intestinal lesion, while it has been recognized that $\mathrm{E}$. coli infrequently ingest red blood cells.

Dey (1974) observed that extensive inhabitants of E. coli within the gut lumen may cause dyspepsia, hyperacidity, gastritis and indigestion. ${ }^{1}$ In addition it has a strong phagocytic activity through which it phagocytosed bacterial flora, fungi such as Sapherita species and even other protozoan parasite such as Giardia lamblia trophozoite. The existence of bacterial flora within or attached to the lumen of mucosa make it more vulnerable to Entamoeba coli attacks. Potassium insufficiency or low potassium is named hypokalemia. Documents split that approximately one out of five people hospitalized in the United States alone have a low potassium level under $3.5 \mathrm{mEq} / \mathrm{L}$. This may engage women with ovarian cysts. It is hypothesized that some ovarian cysts may be a consequence of potassium shortage. There are information that females ages 30-39 have identity-documented to FDA, that they have taken the contraceptive pill, experienced ovarian cysts, and have been found to have low potassium levels. ${ }^{2}$

Pairs in which every partner had an elevated cholesterol level took the longest time to reach pregnancy. Moreover, pairs in which the female had an elevated cholesterol level and the male did not also took longer to attain pregnancy when compared to pairs in which both partners had cholesterol levels in the tolerable range. ${ }^{3}$ Hamad et al showed that; Entamoeba coli infection reduced levels of potassium, phosphorus and raised level of cholesterol. Then we hypothesized that Entamoeba coli is a probable cause of women sub fertility. Extensive work is required to study this neglected microorganism, which is classified up to date as commensal microorganism. I hope to obtain support from institution or international organization to confirm my 
theory. Fund is the corner stone to initiate good research work, which is absent in most of African countries, where research is not a priority to the policy makers.

\section{Acknowledgments}

To editors at MedCrave publishing group for their kind dealing with authors from low income countries.

\section{Conflicts of interest}

The author declares that there are no conflicts of interest.

\section{Funding}

None.

\section{References}

1. http://www.yourarticlelibrary.com/zoology/parasiteentamoeba-coli-lifecycle-mode-of-infection-andtreatment/24270/.

2. https://natural-fertility-info.com/potassium-sodium-for-fertility-health html

3. https://www.nih.gov/news-events/news-releases/nih-study-links-highcholesterol-levels-lower-fertility 